\title{
Numerical study on uncertainty of two-color method
}

\author{
D. Wei \\ weidong@mech.nagaokaut.ac.jp
}

\section{Xiao}

M. Aketagawa

\begin{abstract}
Department of Mechanical Engineering, Nagaoka University of Technology, Nagaoka City, Niigata 940-2188, Japan

School of Mechanical Engineering, Beijing Institute of Technology, Beijing 100081, People's Republic of China

Department of Mechanical Engineering, Nagaoka University of Technology, Nagaoka City, Niigata 940-2188, Japan
\end{abstract}

The two-color method is one of the commonly used approaches for converting a length measured in air to a length in vacuum to eliminate the influence of the refractive index of air. However, the error of the technique is not well known. We investigate this uncertainty based on a generalized expression of the two-color method proposed in this paper and using numerical simulations. Numerical calculations reveal the change of the error with temperature, air pressure, and wavelengths. These characteristics can be used to optimize the two-color method. [DOI: http://dx.doi.org/10.2971/jeos.2015.15051]

Keywords: Two-color method, length measurement, refractive index of air, uncertainty, optimization

\section{INTRODUCTION}

The values of lengths in vacuum are comparable with each other. Because the speed of light in vacuum is constant, the distance of the object from the observation point increases with length. Usually, the length is measured in air is affected by the length in vacuum and the refractive index of air. A larger length measured in air indicates a larger length in vacuum or a smaller refractive index of air. Therefore, we cannot simply compare two lengths in air. Two approaches are used for length conversion to obtain the corresponding lengths in vacuum: one approach is based on precisely measuring the refractive index and the other is based on the application of the two-color method [1] The former utilizes the relationship between the length in vacuum, the length in air, and the refractive index of air. The latter utilizes the relationship between the refractive indices of two different colors. The advantage of the two-color method is that the fluctuations caused by changes of the refractive index can be successfully avoided by using the measured length difference between the two colors.

During length conversion, the uncertainty of the refractive index measurement or the two-color method results in an uncertainty in the estimated value of the length in vacuum. The uncertainty of the calculated refractive index using the empirical formula [2]-[4] is well known. For example, the uncertainty of the Edlén empirical equations $[2,3]$ is on the order of $30-50 \mathrm{~nm} / \mathrm{m}$ [5]. Although the two-color method has been studied by a number of researchers [6]-[12], the uncertainty of the length in vacuum obtained with this technique is not known. We do not know how the order of the uncertainty varies with the measurement environment. The difficulty in the associated uncertainty analysis lies in the fact that two refractive indices used in the two-color method are correlated.
By performing the length measurement using numerical simulations, one can evaluate the error of the two-color method without considering the correlation of two refractive indices. In the present study, we propose a generalized expression of the two-color method and estimate its error using numerical simulations.

\section{METHODS}

In 2009, the national standard of length in Japan changed to a femtosecond optical frequency comb (FOFC). Detailed information about the FOFC can be found in Ref. [13]. The spectrum of an FOFC in the frequency domain can be modeled as a comb function. The comb function is composed of many (generally, more than one million) single-frequency components arranged at intervals equal to the pulse repetition frequency $f_{\text {rep }}$. The entire FOFC is shifted from zero frequency by an offset frequency $f_{C E O}$. A frequency component of an FOFC can be expressed as follows:

$$
f_{P}=(P+Q) \times f_{\text {rep }}=P \times f_{\text {rep }}+f_{C E O},
$$

where the integral part $P$, which is the number of comb lines, is on the order of $10^{6}$, and the fractional part $Q$ is $0 \leq Q<1$.

In general, an FOFC device is synchronized with the coordinated universal time to stabilize its frequency. As can be seen from Eq. (1), if the frequencies of an FOFC are stabilized, the repetition and offset frequencies are also stabilized. According to the definition of the meter, which is based on the fact that the speed of light in vacuum $c_{v a c}$ is constant, the measurement unit $\gamma_{v a c}$ (namely, the wavelength $\lambda_{v a c}$ and the adjacent pulse 
repetition interval length (APRIL) $\delta_{v a c}$ ) can be used to realize the meter as follows [14]:

$$
\gamma_{v a c}=c_{v a c} / f_{P}
$$

Here, $f_{P}$ is the frequency parameter (the frequency $f$ of the wavelength and the pulse repetition frequency $f_{\text {rep }}$ of the APRIL). In this part, the subscripts "vac" and "air" denote the values in vacuum and air, respectively.

The geometric distance $G$ is the true distance between two points in a vacuum. The distances measured using two different wavelengths $\left(\lambda_{\text {air_1 } 1}\right.$ and $\left.\lambda_{\text {air_2 }}\right)$ or APRILs $\left(\delta_{\text {air_1 }}\right.$ and $\left.\delta_{\text {air_2 }}\right)$ in air are the optical distances $L_{\text {rair_1 }}=G / n_{1}$ and $L_{\text {rair_2 }}=G / n_{2} . n$ represents the phase refractive index of air $n_{p}$ at the examined wavelength or the group refractive index of air $n_{g}$ of the APRIL. The estimate $L_{e s t}$ of this geometric distance using the two-color method can be obtained as follows:

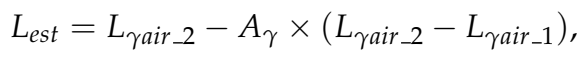

where $\gamma$ denotes the measurement unit and $\mathrm{A}_{\gamma}$ is the so-called $A$-factor defined as follows:

$$
\begin{aligned}
A_{\gamma}= & {\left[n\left(\gamma_{v a c \_2}, T, P, H\right)-1\right] } \\
& /\left[n\left(\gamma_{v a c \_2}, T, P, H\right)-n\left(\gamma_{v a c_{\_} 1}, T, P, H\right)\right] .
\end{aligned}
$$

Here, $T, P$, and $H$ are the temperature, barometric pressure, and humidity, respectively.

By applying the law of propagation of uncertainty $[15,16]$ to Eq. (3), we have

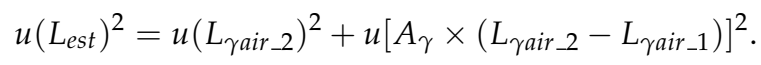

Here, $u(x)$ denotes the uncertainty of variable $x$. The uncertainty of the first term of the right-hand side of Eq. (5) is

$$
\left[u\left(L_{\text {rair_2 }}\right) / L_{\text {rair_2 }}\right]^{2}=\left[u\left(n_{2}\right) / n_{2}\right]^{2} .
$$

The uncertainty of the second term of the right-hand side of Eq. (5) is

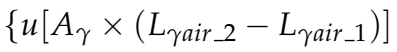

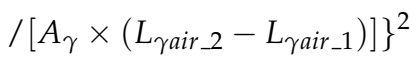

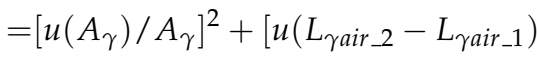

$$
\begin{aligned}
& \left./\left(L_{\text {rair_2 }}-L_{\text {rair_1 } 1}\right)\right]^{2} \text {. }
\end{aligned}
$$

Based on Eq. (4), the uncertainty of the first term of the righthand side of Eq. (7) is

$$
\begin{aligned}
& {\left[u\left(A_{\gamma}\right) / A_{\gamma}\right]^{2} } \\
= & \left\{\frac{u\left[n\left(\gamma_{v a c_{\_} 2}, T, P, H\right)-1\right]}{n\left(\gamma_{v a c \_2}, T, P, H\right)-1}\right\}^{2} \\
& +\left\{\frac{u\left[n\left(\gamma_{v a c \_2}, T, P, H\right)-n\left(\gamma_{v a c_{\_} 1}, T, P, H\right)\right]}{n\left(\gamma_{v a c \_2}, T, P, H\right)-n\left(\gamma_{v a c_{-} 1}, T, P, H\right)}\right\}^{2} \\
& +\frac{u\left[n\left(\gamma_{v a c \_2}, T, P, H\right)\right]}{\left[n\left(\gamma_{v a c \_2}, T, P, H\right)-1\right]} \\
& \times \frac{\left\{u\left[n\left(\gamma_{v a c \_2}, T, P, H\right)\right]-u\left[n\left(\gamma_{v a c_{-} 1}, T, P, H\right)\right]\right\}}{\left[n\left(\gamma_{v a c \_2}, T, P, H\right)-n\left(\gamma_{v a c_{-} 1}, T, P, H\right)\right]} .
\end{aligned}
$$

The uncertainty of the first term of the right-hand side of Eq. (8) is

$$
\left\{u\left[n\left(\gamma_{v a c_{2} 2}, T, P, H\right)-1\right]\right\}^{2}=u\left[n\left(\gamma_{v a c \_2}, T, P, H\right)\right]^{2} .
$$

The uncertainty of the second term of the right-hand side of Eq. (8) is

$$
\begin{aligned}
& \left\{u\left[n\left(\gamma_{v a c_{2} 2}, T, P, H\right)-n\left(\gamma_{v a c_{-} 1}, T, P, H\right)\right]\right\}^{2} \\
= & u\left[n\left(\gamma_{v a c_{-} 2}, T, P, H\right)\right]^{2}+u\left[n\left(\gamma_{v a c_{1} 1}, T, P, H\right)\right]^{2} \\
& +2 \times u\left[n\left(\gamma_{v a c_{2} 2}, T, P, H\right)\right] \times u\left[n\left(\gamma_{v a c_{-} 1}, T, P, H\right)\right] \\
& \times \alpha\left(\gamma_{v a c_{\_} 1}, \gamma_{v a c_{-} 2}, T, P, H\right) .
\end{aligned}
$$

In Eq. $(10), \alpha\left(\gamma_{v a c_{-} 1}, \gamma_{v a c \_}, T, P, H\right)$ is the correlation coefficient used to characterize the degree of correlation between $u\left[n\left(\gamma_{v a c_{-} 2}, T, P, H\right)\right]$ and $u\left[n\left(\gamma_{v a c_{-} 1}, T, P, H\right)\right]$. $\alpha\left(\gamma_{v a c_{-} 1}, \gamma_{v a c_{2} 2}, T, P, H\right)$ is defined as follows:

$$
\begin{aligned}
& \alpha\left(\gamma_{v a c_{\_} 1}, \gamma_{v a c_{\_} 2}, T, P, H\right) \\
= & \frac{u\left[n\left(\gamma_{v a c_{-} 1}, T, P, H\right) \times n\left(\gamma_{v a c \_2}, T, P, H\right)\right]}{u\left[n\left(\gamma_{v a c \_2}, T, P, H\right)\right] \times u\left[n\left(\gamma_{v a c_{-} 1}, T, P, H\right)\right]} .
\end{aligned}
$$

The uncertainty of the second term of the right-hand side of Eq. (7) is also the function of $u\left[n\left(\gamma_{v a c \_}, T, P, H\right)\right]$ and $u\left[n\left(\gamma_{v a c_{-} 1}, T, P, H\right)\right]$. That means its uncertainty is affected by the correlation coefficient $\alpha\left(\gamma_{v a c_{-} 1}, \gamma_{v a c_{2}}, T, P, H\right)$. The estimation of the correlation coefficient $\alpha\left(\gamma_{v a c_{-} 1}, \gamma_{v a c_{-} 2}, T, P, H\right)$ is difficult. In addition, as seen above, it is complicated to estimate the uncertainty of the two-color method based on the law of propagation of uncertainty. To solve this problem, by performing the numerical simulations, we evaluate the error of the two-color method without considering the correlation coefficient $\alpha\left(\gamma_{v a c_{-} 1}, \gamma_{v a c_{2} 2}, T, P, H\right)$.

The calculation is performed as follows. First, we set a geometric length $G_{s e t}$. Then, we specify the environmental parameters $(T, P, H)$. Based on Eq. (3) and (4), we calculate a value $L_{e s t}$, which is estimated using the two-color method. Finally, we treat $L_{e s t}-G_{\text {set }}$ as the error of the two-color method.

Before discussing the numerical simulations, we note the difference between the above expressions and the previously proposed two-color methods [1], [6]-[12]. The latter are related to either the wavelength or the APRIL. The former are generalized expressions that can be used for length conversion in which not only the wavelength but also the APRIL can be used as a scale.

\section{RESULTS AND DISCUSSION}

We abbreviate all instances of "wavelengths" or "center wavelengths of the APRILs" with "WLs" or "CWAs," respectively.

The WLs and CWAs used in the calculations were $1560 \mathrm{~nm}$ and $780 \mathrm{~nm}$, respectively. The value of $G_{s e t}$ was $1 \mathrm{~m}$. The calculations were performed under standard environmental conditions (temperature of $20^{\circ} \mathrm{C}$, pressure of $101.325 \mathrm{kPa}$, and $0 \%$ humidity). The $0 \%$ humidity was selected based on previous studies [7]-[9], [17]-[21] that showed that the length $L_{\text {est }}$ can be determined with optimum precision when the humidity is $0 \%$. To calculate $n_{p}$, we used the Edlén empirical equations, given in Ref. [5]. The calculation procedures for $n_{g}$ are described in Ref. [14]. The lengths obtained using different colors in a specific environment were calculated using $G_{s e t} / n$. Based

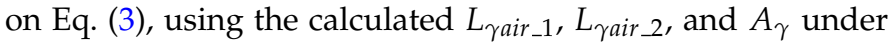




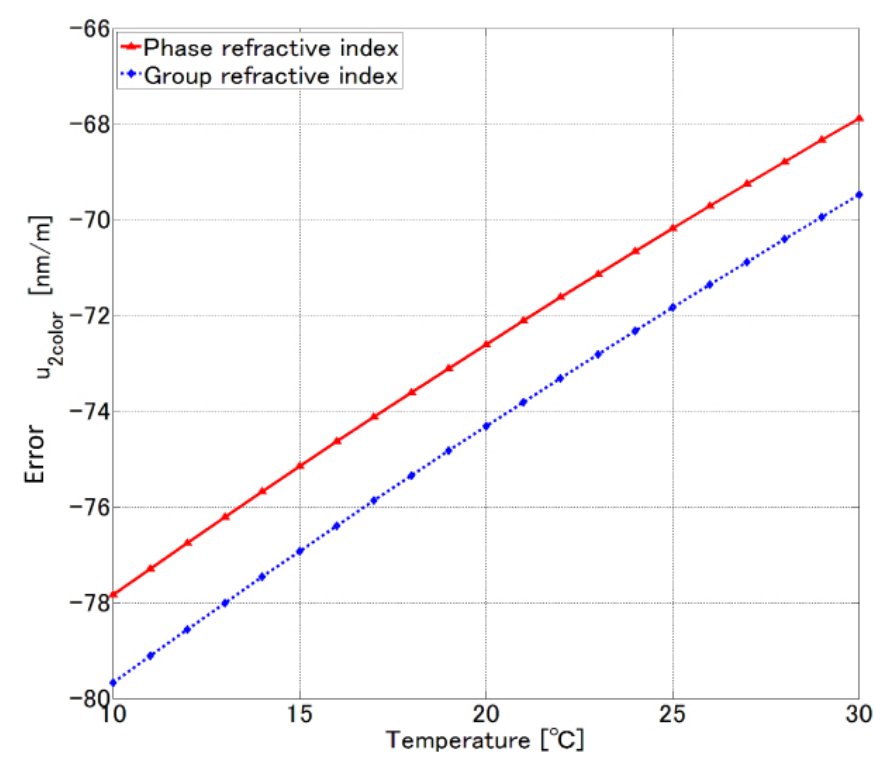

FIG. 1 Error of the two-color method $u_{2 \text { color }}$ as a function of temperature for the phase refractive index of air $n_{p}$ (triangle and solid line) and group refractive index of air $n_{g}$ (plus sign and dotted line).

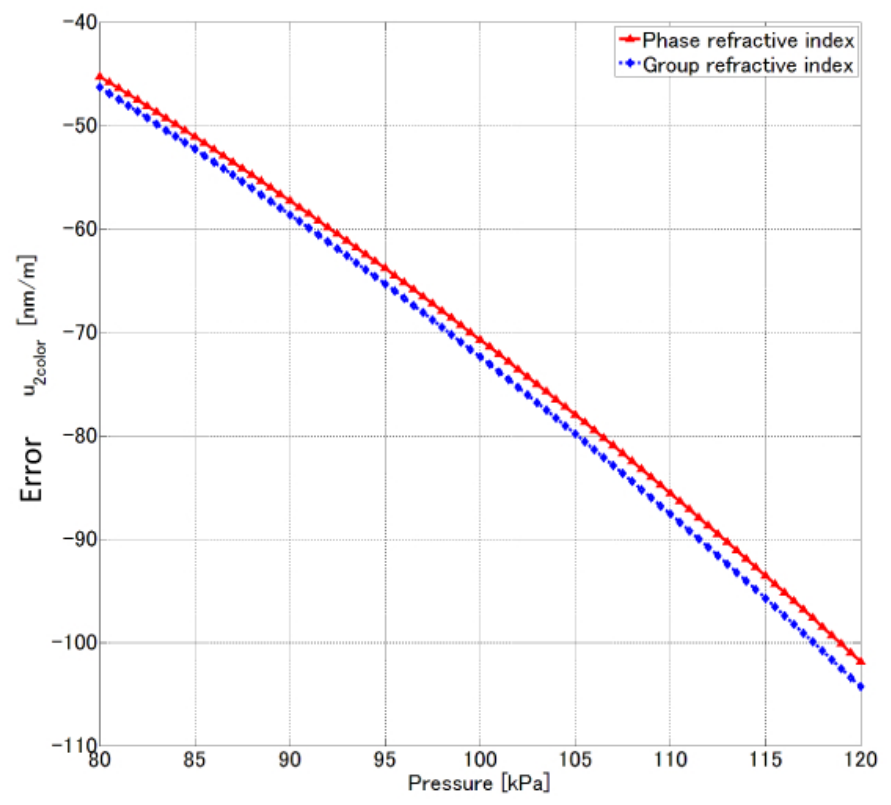

FIG. 2 Error of the two-color method $u_{2 \text { color }}$ as a function of air pressure for the phase refractive index of air $n_{p}$ (triangle and solid line) and the group refractive index of air $n_{g}$ (plus sign and dotted line).

standard environment, we estimated the length in vacuum and considered the difference between the estimated length $L_{\text {est }}$ and the set length $G_{\text {set }}$ to be the error of two-color method $u_{2 \text { color }}$.

Figures 1 and 2 show the variation in $u_{2 \text { color }}$ when the environmental parameters change within realistic ranges $\left(T \in[10,30]{ }^{\circ} \mathrm{C}, P=101.325 \mathrm{kPa}, H=0 \%\right)$. and $\left(T={ }^{\circ} \mathrm{C}\right.$, $P \in[80,120] \mathrm{kPa}, H=0 \%)$. As shown in Figure $1, u_{2 \text { color }}$ is reduced with the increase of the temperature; however, it increases with the increase of air pressure, as shown in Figure 2.
Tables 1 and 2 display $u_{2 \text { color }}$ for both refractive indices under realistic environmental conditions $\left(T \in[10,30]{ }^{\circ} \mathrm{C}\right.$, $P \in[80,120] \mathrm{kPa}, H=0 \%)$. The tables show that the minimum and maximum $u_{2 \text { color }}$ values are obtained for a temperature of $30^{\circ} \mathrm{C}$ and a pressure of $80 \mathrm{kPa}$, and a temperature of $10^{\circ} \mathrm{C}$ and a pressure of $120 \mathrm{kPa}$, respectively.

\begin{tabular}{|c|c|c|c|}
\hline Pressure $[\mathrm{kPa}]$ & \multicolumn{3}{|c|}{ Temperature $\left[{ }^{\circ} \mathrm{C}\right]$} \\
& 10 & 20 & 30 \\
\hline 80.000 & -48.5 & -45.3 & -42.3 \\
\hline 101.325 & -77.8 & -72.6 & -67.9 \\
\hline 120.000 & -109.2 & -101.8 & -95.2 \\
\hline
\end{tabular}

TABLE 1 Error of the two-color method $u_{2 \text { color }}$ calculated using the phase refractive index of air $n_{p}$ for different environmental conditions.

\begin{tabular}{|c|c|c|c|}
\hline Pressure $[\mathrm{kPa}]$ & \multicolumn{3}{|c|}{ Temperature $\left[{ }^{\circ} \mathrm{C}\right]$} \\
& 10 & 20 & 30 \\
\hline 80.000 & -49.7 & -46.3 & -43.3 \\
\hline 101.325 & -79.7 & -73.4 & -69.5 \\
\hline 120.000 & -111.8 & 104.2 & -97.5 \\
\hline
\end{tabular}

TABLE 2 Error of the two-color method $u_{2 \text { color }}$ calculated using the group refractive index of air $n_{g}$ for different environmental conditions.

Figure 3 shows the variation of $u_{2 \text { color }}$ due to a shift in $G_{s e t}$ under standard environmental conditions. $u_{2 \text { color }}$ is directly proportional to $G_{s e t}$. The slopes of the lines are $-72.6 \mathrm{~nm} / \mathrm{m}$ for $n_{p}$ and $-73.4 \mathrm{~nm} / \mathrm{m}$ for $n_{g}$.

Note that $u_{2 \text { color }}$ is affected by the WLs or CWAs used. Hence, we examined the changes in $u_{2 \text { color }}$ by varying the WL or CWA. The range of WLs or CWAs used in the numerical simulations corresponds to the currently provided length standard in Japan, which is in the range of 500-1684 nm. Generally, the relationship between the WLs or CWAs in the twocolor method is associated with a fundamental wave ${ }^{-}$and its second harmonic $\lambda / 2$. The WLs or CWAs of the fundamental wave were found to be in the range of 1000-1684 nm and those of the second harmonic wave were in the range of 500$842 \mathrm{~nm}$. Figure 4 shows $u_{2 \text { color }}$ as a function of WL or CWA of the fundamental wave in vacuum. When the WL or CWA increased, $u_{2 \text { color }}$ decreased.

As can be seen in Figure 4, under standard environmental conditions, the minimum achievable $u_{2 \text { color }}$ corresponds to the 842 $\mathrm{nm}$ and $1684 \mathrm{~nm}$ pair. From Table 1 , the minimum $u_{2 \text { color }}$ is obtained for $30^{\circ} \mathrm{C}$ and $80 \mathrm{kPa}$. In these conditions, $u_{2 \text { color }}$ of the $842 \mathrm{~nm}$ and $1684 \mathrm{~nm}$ pair was calculated as $-42.2 \mathrm{~nm} / \mathrm{m}$ using $n_{p}$ and $-43.1 \mathrm{~nm} / \mathrm{m}$ using $n_{g}$.

As can be seen in Figure 4, under standard environmental conditions, the maximum $u_{2 \text { color }}$ was obtained for the $500 \mathrm{~nm}$ and $1000 \mathrm{~nm}$ pair. According to Table 2, the maximum $u_{2 \text { color }}$ is obtained for $10{ }^{\circ} \mathrm{C}$ and $120 \mathrm{kPa}$. In these conditions, $u_{2 \text { color }}$ was calculated as $-111.1 \mathrm{~nm} / \mathrm{m}$ for $n_{p}$ and $-117.6 \mathrm{~nm} / \mathrm{m}$ for $n_{g}$.

We can conclude that by using WLs or CWAs within the 500-1684 nm range under realistic environmental condi- 


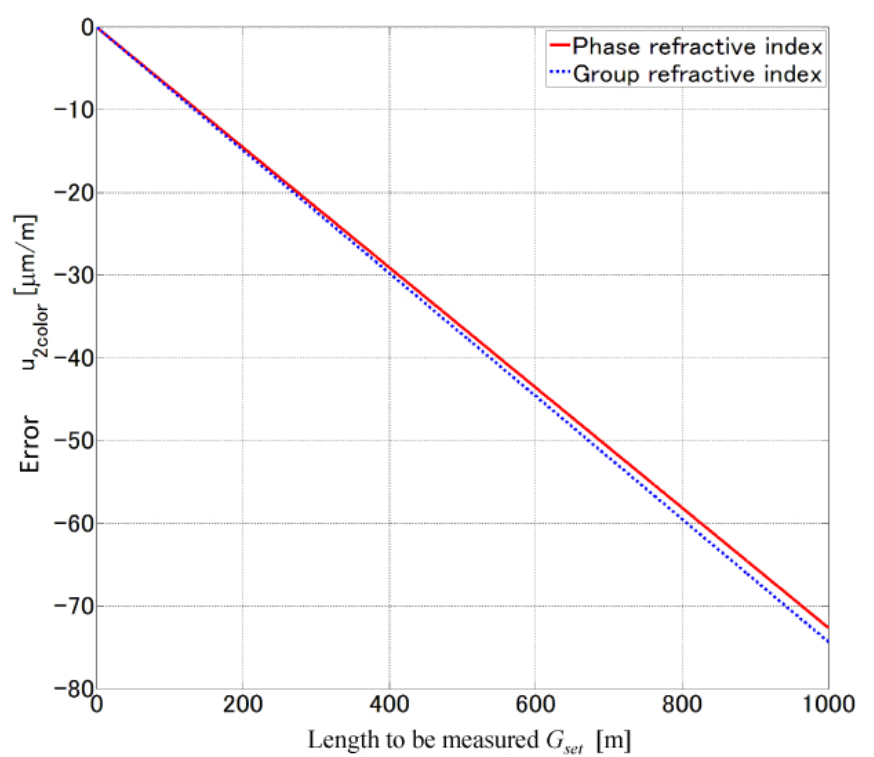

FIG. 3 Error of the two-color method $u_{2 \text { color }}$ as a function of the length to be measured $G_{\text {set }}$.

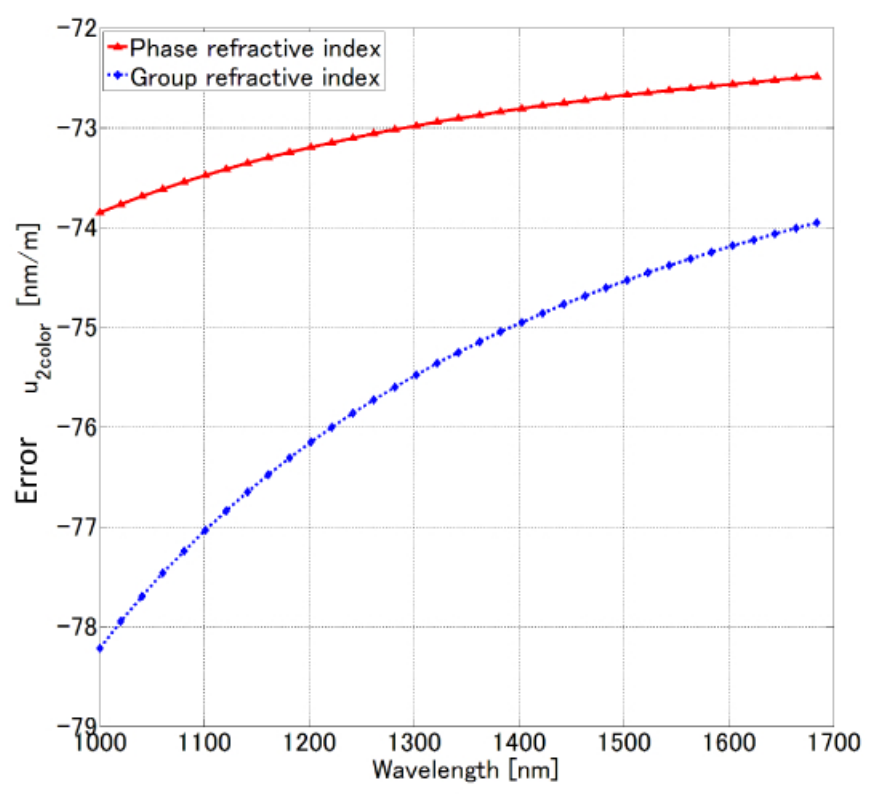

FIG. 4 Error of the two-color method $u_{2 \text { color }}$ as a function of WL or CWA in vacuum.

tions, the achievable $u_{2 \text { color }}$ is in the range of $-42.2 \mathrm{~nm} / \mathrm{m}$ to $-111.1 \mathrm{~nm} / \mathrm{m}$ for $n_{p}$ and $-43.1 \mathrm{~nm} / \mathrm{m}$ to $-117.6 \mathrm{~nm} / \mathrm{m}$ for $n_{g}$.

We also compare the two-color method and the techniques based on the Edlén empirical equations. The method using the empirical equations is a passive approach, because measuring environmental parameters is a passive activity. The two-color method can be used to make an interferometer less sensitive to environmental conditions by using two WLs or CWAs. Here, we show the change of $u_{2 \text { color }}$ due to a change in environmental parameters. The results show the possibility of obtaining a smaller $u_{2 \text { color }}$ by controlling the environmental parameters in laboratory conditions or selecting preferred conditions in open-air fields. Hence, the two-color method can be used as an active approach to compensate the influence of the refractive index of air. With further optimization, the two-color method can be used to obtain measurements with a smaller error than that of the empirical equations.

\section{CONCLUSION}

We proposed a generalized expression of the two-color method for length conversion, in which not only the wavelength but also the APRIL can be used as a scale. Using numerical simulations in a realistic environmental parameter range $\left(T \in[10,30]{ }^{\circ} \mathrm{C}, P \in[80,120] \mathrm{kPa}, H=0 \%\right)$, we found out for the first time that the achievable errors are in the range of $-42.2 \mathrm{~nm} / \mathrm{m}$ to $-111.1 \mathrm{~nm} / \mathrm{m}$ for the $n_{p}$ and $-43.1 \mathrm{~nm} / \mathrm{m}$ to $-117.6 \mathrm{~nm} / \mathrm{m}$ for the $n_{g}$ calculations. We also showed the change of the error with temperature, air pressure, and wavelength, which is useful to obtain the smallest error from an active point of view. The findings of this study provide a better insight into the two-color method, which will increase the opportunity to apply this method in various length-measurement applications.

\section{ACKNOWLEDGEMENTS}

This research work was partially financially supported by a grant from the Mitutoyo Association for Science and Technology (MAST).

\section{References}

[1] P. L. Bender, and J. C. Owens, "Correction of Optical Distance Measurements for the Fluctuating Atmospheric Index of Refraction," J. Ceophys. Res. 70, 2461-2462 (1965).

[2] E. Bengt, "The Refractive Index of Air," Metrologia 2, 71 (1966).

[3] K. P. Birch, and M. J. Downs, “An Updated Edlén Equation for the Refractive Index of Air," Metrologia 30, 155 (1993).

[4] P. E. Ciddor, "Refractive index of air: new equations for the visible and near infrared," Appl. Optics 35, 1566-1573 (1996).

[5] J. A. Stone, and J. H. Zimmerman, "Refractive index of air calculator," http://emtoolbox.nist.gov/Wavelength/Edlen.asp.

[6] B. Querzola, "High accuracy distance measurement by twowavelength pulsed laser sources," Appl. Optics 18, 3035-3047 (1979).

[7] H. Matsumoto, and T. Honda, "High-accuracy length-measuring interferometer using the two-colour method of compensating for the refractive index of air," Meas. Sci. Technol. 3, 1084 (1992).

[8] K. Minoshima, and H. Matsumoto, "High-Accuracy Measurement of $240-\mathrm{m}$ Distance in an Optical Tunnel by use of a Compact Femtosecond Laser," Appl. Optics 39, 5512-5517 (2000).

[9] K. Minoshima, K. Arai, and H. Inaba, “High-accuracy self-correction of refractive index of air using two-color interferometry of optical frequency combs," Opt. Express 19, 26095-26105 (2011).

[10] G. Wu, M. Takahashi, K. Arai, H. Inaba, and K. Minoshima, "Extremely high-accuracy correction of air refractive index using twocolour optical frequency combs," Sci. Rep. 3, 1894 (2013).

[11] D. Wei, M. Aketagawa, K. Takamasu, and H. Matsumoto, "Twocolor absolute length measuring method based on pulse repetition interval lengths," Opt. Eng. 53, 122413 (2014). 
[12] D. Wei, and M. Aketagawa, "Comparison of two-color methods based on wavelength and adjacent pulse repetition interval length," J. Eur. Opt. Soc.-Rapid 9, 14031 (2014).

[13] J. Ye, and S. T. Cundiff, Femtosecond optical frequency comb: principle, operation, and applications (Springer, New York, 2005).

[14] D. Wei, and M. Aketagawa, "Uncertainty in length conversion due to change of sensitivity coefficients of refractive index," Opt. Commun. 345, 67-70 (2015).

[15] H. W. Coleman, and W. G. Steele, Experimentation and Uncertainty Analysis for Engineers (Wiley, Haboken, 1999).

[16] B. Walter, G. C. Maurice, and M. H. Peter, "Evolution of the 'Guide to the Expression of Uncertainty in Measurement'," Metrologia 43, S161 (2006).

[17] A. Ishida, "Two-Wavelength Displacement-Measuring Interferometer Using Second-Harmonic Light to Eliminate Air-TurbulenceInduced Errors," Jpn. J. Appl. Phys. 28, L473 (1989).
[18] H. Matsumoto, Y. Zhu, S. Iwasaki, and T. Oishi, "Measurement of the changes in air refractive index and distance by means of a two-color interferometer," Appl. Optics 31, 4522-4526 (1992).

[19] A. N. Golubev, and A. M. Chekhovsky, "Three-color optical range finding," Appl. Optics 33, 7511-7517 (1994).

[20] K. Meiners-Hagen, and A. Abou-Zeid, "Refractive index determination in length measurement by two-colour interferometry," Meas. Sci. Technol. 19, 084004 (2008).

[21] G. Wu, K. Arai, M. Takahashi, H. Inaba, and K. Minoshima, "Highaccuracy correction of air refractive index by using two-color heterodyne interferometry of optical frequency combs," Meas. Sci. Technol. 24, 015203 (2013). 